\title{
Mejores prácticas en gestión de proveedores a nivel mundial, aplicables al sistema de Administración de la Relación con el Proveedor (ARP) de la Jefatura de Operaciones Logísticas (JOL) de la Fuerza Aérea Colombiana (FAC)'
}

Best Practices in Supplier Management Worldwide, Applicable

to Supplier Relationship Management (SRM) System of the Head of Logistics Operations of the Colombian Air Force ${ }^{2}$

Italo Arrazola Aguado 3

CIENCIA Y PODER AÉREO ISSN 1909-7050 | E-ISSN 2389-2468 |Volumen 9 | Enero-Diciembre de 2014 | Colombia | Pp. 33-41

Recibido: 11/11/2014 Aprobado evaluador interno: 18/11/2014 Aprobado evaluador externo: 25/11/2014

'Artículo de revisión para optar al título de Especialista en Logística Aeronáutica.

2 Review article as partial fullfilment of the requirements for the degree of specialist in Aeronautical logistics.

${ }^{3}$ Administrador Aeronáutico de la Fuerza Aérea Colombiana, Ingeniero Químico de la Universidad de Antioquia. Mayor de la Fuerza Aérea Colombiana. Director del Centro de Investigación en Tecnologías Aeroespaciales - CITAE. Correo electrónico: italo.arrazola@fac.mil.co

Aeronautical Administrator from the Colombian Air Force, Chemical Engineer from the Antioquia University. Major of the Colombian Air Force. Director of the Research Centre for Aerospace Technologies - CITAE.E-mail: italo.arrazola@fac.mil.co
Resumen: Dentro de la administración de la cadena de abastecimiento ó Supply Chain Management (SCM), se destaca la gestión de proveedores como una de las actividades más críticas para su establecimiento efectivo. Por lo anterior, el presente artículo busca aproximarse al estado del arte en gestión de proveedores, utilizando como referencia revistas internacionales especializadas en el tema, ofreciendo así una base de referencia en futuras investigaciones relacionadas. En este sentido, se propone un conjunto de alternativas para estructurar un procedimiento general, que permita la adecuada toma de decisiones en cuanto a los proveedores tanto en la Jefatura de Operaciones Logísticas de la Fuerza Aérea Colombiana, como en cualquier organización con el ajuste apropiado.

Palabras clave: Administración, ARP, cliente, proveedor, relación.

Abstract: Nowadays, the Supply Chain Management (SCM) is of the utmost importance, standing inside this the supplier management as one of the most critical activities for its effective implementation. Therefore, this article aims to approach the state of the art in supplier management, using international journals as the reference point, providing a baseline for future related research in the matter. In this sense a set of alternatives is proposed to structure a general procedure for appropriate decisions making, regarding suppliers in the Head of Logistics Operations of the Colombian Air Force, as in any organization with the proper fit.

Key Words: Customer, Management, Relationship, SRM, Supplier. 


\section{Introducción}

Para el primer decenio del siglo XXI, la administración de la cadena de suministros o Supply Chain Management (SCM) reviste la mayor importancia, destacándose como uno de los principales objetos de estudio dentro de la administración a nivel mundial y dentro de ésta la selección de proveedores se constituye en una de las actividades más críticas para su establecimiento efectivo, afectando el desempeño de las organizaciones ya que las compras llegan en algunas ocasiones a representar hasta el 70\% del costo mismo del producto, teniendo el departamento de compras una gran importancia en la reducción de los costos totales de producción (Barla, 2003; Aksoy y Öztük, 2011; Yeung, 2008), debiendo buscar estrategias para la alineación de los objetivos de compra con los objetivos de la organización, exigiendo un papel más proactivo por parte de la empresa en la selección de proveedores y su evaluación continua (Pressey, Winklhofer, y Tzokas, 2009), como lo expresan Oly, Muhamad, Loo Cha, y Salleh (2005), utilizar la estrategia correcta para la selección y administración de los proveedores asegura la escogencia justa de proveedores que puedan soportar adecuadamente la producción, siendo lo suficientemente flexibles en cuanto a factores de producto, lanzamiento y volumen para cumplir con las fluctuantes necesidades de los clientes.

Como es ya ampliamente conocido, debido a la globalización las empresas en la actualidad encuentran abastecimiento en el punto geográfico que les ofrezca ventajas de posición y costos, pudiendo fabricar sus productos en un punto geográfico completamente diferente para disminuir costos de operación y finalmente, distribuir su producto a la mayor cantidad de mercados posibles y económicamente viables. Por lo tanto, para una empresa que desee acceder a las ventajas de la globalización, el desarrollo de estrategias que agreguen valor y reduzcan costos son fundamentales, siendo entonces necesaria la adopción de prácticas de gestión colaborativa, que requieren una adecuada selección y evaluación de proveedores, gestionando la triada abastecimiento-fabricación-distribución bajo el concepto de administración de la cadena de suministros, buscando su integración coordinada (Sarache Castro, Castrillón Gómez, y Ortiz Franco, 2009).

\section{Objetivos}

El objetivo general del artículo es establecer el procedimiento general con las opciones de herramientas adecuadas que permitan estructurar un sistema de ad- ministración de la relación con el proveedor (ARP) aplicable a la JOL de la FAC, que conlleve al cumplimiento de los requerimientos en logística aeronáutica en cuanto a tiempos de entrega, trazabilidad, calidad y costos por parte de los proveedores.

\section{Objetivos específicos}

- Realizar una revisión general de las relaciones en la industria global entre proveedores y clientes dentro de la administración de cadena de suministros, describiendo las ventajas y posibles desventajas de la aplicación de los sistemas encontrados.

- Describir el mejor sistema de administración de la relación con el proveedor aplicable al sector aeronáutico.

- Proponer el sistema que por sus características sea aplicable a las relaciones entre la Jefatura de Operaciones Logísticas Aeronáuticas de la FAC y sus proveedores.

\section{Metodología}

Para la elaboración del artículo se realizó una investigación de enfoque cualitativo de tipo descriptivo, mediante una revisión bibliográfica de más de 50 artículos indexados como base para un análisis individual del autor, haciendo una aproximación a la gestión de proveedores.

\section{Relación con los proveedores dentro de la SCM}

Las diferentes relaciones con los proveedores dentro de la cadena de suministros, es posible dividirlas en dos grandes enfoques o sistemas. Uno es el que acepta la ineficiencia del proveedor y busca modelos que le permitan administrarla para poder minimizarla, como el modelo matemático de Ruiz, Ablanedo y Ayala (2012), donde se asignan valores de probabilidad a las variables de incumplimiento en la entrega, flexibilidad del proveedor para responder ante un nuevo pedido y costos asociados con las diferentes prioridades de compra (regular, emergencia, pérdida), buscando la mejor distribución en las órdenes de compra que permitan flexibilidad y agilidad en la respuesta ante las eventualidades previstas.

El segundo enfoque es el de transformar el antiguo sistema de relación simple cliente-proveedores en un 
sistema colaborativo, donde se requiere un desempeño superior por parte del proveedor para lograr los objetivos, como resultado de la integración y cooperación de la organización y entre las organizaciones de la cadena de suministros, que son ideas fundamentales en la gestión moderna, con varias opciones de colaboración cliente-proveedor (Eliacy Cavalcanti y Alexandre Tadeu, 2013), entre ellas se encuentran las siguientes:

- Negociación: acuerdos sobre precios y relaciones con la competencia.

- Cooperación: pocos proveedores y contratos a largo plazo.

- Coordinación: conexiones vía Tecnologías de la Información y de la Comunicación TIC) y compartir información.

- Colaboración: integración de la cadena de suministros; planeamiento conjunto y compartir tecnología.

Cada una de las opciones de colaboración requiere un nivel diferente de interrelación cliente-proveedor siendo la última la de mayor nivel, la cual es utilizada en el área de la cadena de producción automotriz, constituyéndose en un gran ejemplo donde el sistema colaborativo implica un apoyo mutuo cliente proveedor, donde las empresas líderes en fabricación de automóviles brindan el know-how y la asistencia necesaria para que el proveedor pueda certificarse e integrarse en su cadena de valor, (Arroyo López y Cárcamo Solis, 2009) para lograr un crecimiento sinérgico de cooperación, compartiendo recursos y habilidades para alcanzar beneficios conjuntos con resultados de éxito para todos los intervinientes, que de manera individual seguramente no lo conseguirían (Slack, Chambers, y Johnston, 2009).

\section{Sistema simple vs. Sistema colaborativo}

El sistema simple es el que se utiliza para la contratación estatal en Colombia mediante la Ley 80 de 1993 que resalta el principio de economía en sus artículos 21 y 25 como criterio de selección, que en el subtítulo "selección y evaluación de proveedores" del presente artículo se define como de nivel 1, donde los criterios de selección de proveedores son básicamente precio y calidad sin ningún tipo de compromiso o confianza mutua, lo que lleva en muchas ocasiones a costos adicionales posteriores debidos a incumplimiento por parte del proveedor, lo que afecta la producción y a su vez la confianza del siguiente cliente dentro de la cadena de suministros (Mazzali, Machado Junior, y Jaciara Furlaneto, 2011); mientras el sistema colaborativo ofrece una innegable estabilidad y cumplimiento de los criterios establecidos pero a un plazo y costos inmediatos significativamente mayores, vistos desde el punto de vista de proceso, pero si se mantiene el esquema de SCM se encuentra que al alcanzar la estabilidad se tiene un costo total menor (Partida, 2012).

Claro es entonces entre los sistemas mencionados, que como en cualquier otro aspecto de la vida diaria, el de mejores y más sólidos resultados es el que requiere a su vez un mayor esfuerzo, ya que lograr un sistema colaborativo requiere un gran entendimiento y confianza mutuas en el proceso de adquisiciones, que en el ámbito empresarial se conoce como comakership, siendo la confianza el factor clave para el desarrollo de asociaciones entre los diferentes agentes de la cadena de suministros, que a su vez está relacionada con un contexto cultural propio de cada región del planeta (Dyer y Chu, 2003; Zaheer y Zaheer, 2006; Marques Vieira, Laureano Paiva, Beheregarai Finger, y Teixeira, 2013).

Una relación colaborativa entre proveedor y cliente es uno de los componentes más difíciles de lograr, ya que esta relación no se limita a una simple alianza, sino a la mencionada confianza mutua, la cual no se construye de la noche a la mañana, precisa tiempo, un largo plazo que permita una construcción de vínculos profundos (Hines, 2004), incrementando a su vez la importancia de la adecuada selección de proveedores, debiendo incluir factores cualitativos que dificultan el establecimiento de los criterios de selección y evaluación (Ghodsypour y O'Brien, 1998). Este es el tipo de relación que se busca alcanzar en la SCM, ya que la utilización y dependencia en proveedores es simplemente una necesidad de las organizaciones productivas, debido a que ninguna posee la tecnología y capacidad de producirlo todo (Kannan y Tan, 2002) y las relaciones colaborativas a largo plazo conducen siempre al éxito tanto operacional como financiero (Pimentel Claro, Borin de Oliveira Claro, y Zylbersztajn, 2005).

Las relaciones colaborativas para entidades de seguridad nacional como la FAC requieren de atención especial, debido a que las mejoras en el desempeño que requieren compartir información de pronósticos, planeamiento, capacidad estructural y ubicación (Vereecke y Muylle, 2006), se constituyen en decisiones que afectan la seguridad nacional, sobre todo cuando los proveedores son de origen extranjero. 


\section{Selección del sistema}

Teniendo en cuenta que como lo expone Partida (2012), el adoptar y mantener un sistema colaborativo requiere inversión adicional de recursos durante la selección y el desarrollo de los proveedores, se puede inferir que no todos los proveedores pueden o requieren ser desarrollados en la FAC como en cualquier otra empresa o institución, sino desde un punto de vista práctico aquellos que provean lo que signifique un alto riesgo para el cumplimiento de los requerimientos del cliente, ya que de lo contrario se requeriría de una gran inversión de recursos de la empresa (tiempo, inversiones, personal, entre otros.). Es por esto, que se debe determinar la importancia de cada uno de los suministros dentro de la SCM para poder así organizarlos por categorías que permitan establecer cuál de los sistemas es más apropiado para la gestión de sus proveedores, permitiendo enfocar el esfuerzo y los recursos a aquellas categorías que posean una ponderación importante dentro de los criterios establecidos por la organización. Este manejo de proveedores por categorías se conoce como Category Management (CM), cuya esencia es la de crear valor para la compañía a través del apalancamiento o utilización de recursos y capacidades externas, reduciendo ineficiencias a través del planeamiento colaborativo y la utilización de recursos comunes (Monczka y Markham, 2014; Rashid y Matilla, 2011).

El CM para industrias que tienen baja demanda comparado con la capacidad de producción de los fabricantes (como la FAC), reviste de una mayor importancia, ya que no se debe limitar a una aproximación comprador-vendedor, sino a una relación desde un punto de vista SCM, llevando al equipo de compras a ser líder de categoría, lo que significa desarrollar un conocimiento detallado del proveedor (O'Keeffe y fearne, 2002), que permita ajustar el planeamiento a las capacidades y etapas de producción y respuesta de las casas fabricantes, o en los casos imprevistos al modelo de negocios de los intermediarios. Se trata de introducir innovaciones en los procesos basados en una combinación de conocimientos y experiencia de cada integrante de la cadena de suministros tanto individual como en conjunto (Duanmu y Fai, 2007).

\section{Selección y evaluación de proveedores}

Para cualquier tipo de relación con los proveedores que se escoja, se requiere una selección inicial, a partir de la cual se comienza la gestión de dichos proveedores, así como una evaluación periódica posterior que permita definir ajustes en el nivel de integración ó su permanencia en el sistema.

Las variables y el tamaño de la base de proveedores requerido para la selección inicial, dependerán del enfoque escogido de lo mencionado en el subtítulo anterior, ya que no es igual seleccionar calculando fallas dentro del proceso que hacerlo con el fin de construir vínculos a largo plazo. Como lo exponen Huang y Keskar (2007) el elemento fundamental para el éxito de un proceso de selección de proveedores es la cuidadosa determinación de los criterios de evaluación que responden tanto a la estrategia como a los objetivos de la organización, debiendo ser continuamente revisados y actualizados.

Para la determinación adecuada de los criterios se debe establecer el nivel de integración cliente-proveedor siendo una excelente guía la definida por Ghodsypour y O'brien (1998), quienes propusieron que los criterios para selección de proveedores dependen de la competitividad de la empresa, su estrategia corporativa y el nivel de integración comprador-proveedor buscado, siendo esos niveles los siguientes:

- Nivel 1: en este nivel no hay ninguna integración. Los criterios más importantes son precio y calidad.

- Nivel 2: en este nivel existe una integración logística entre comprador y proveedor, teniendo el proveedor un rol importante en la competitividad del comprador. En estas circunstancias al precio y la calidad se adicionan elementos logísticos operacionales como confiabilidad, flexibilidad, capacidad de producción, lead time y los demás que puedan ser identificados dentro de las necesidades de abastecimiento.

- $\quad$ Nivel 3: en este nivel se busca una integración operacional entre comprador y proveedor. Es el usualmente escogido para la implementación de un sistema de abastecimiento Just in Time (JIT), por lo tanto ya no solo es importante tener en cuenta las características de salida del proveedor, sino la manera en que dichos servicios son provistos, en otras palabras se considera como criterios de selección adicionales a los niveles anteriores los índices de capacidad del proceso del proveedor, como tiempos de producción, tamaño de lote, entre otros. 
- Nivel 4: la integración para esta situación es más profunda que en los niveles anteriores, y se realiza cuando la administración corporativa decide integrar tanto su proceso como su producto con el proveedor. A los criterios del nivel anterior se suman características de recursos humanos del proveedor desde varios puntos de vista, como participación en el diseño, capacidad administrativa, cultura organizacional, etc.

- $\quad$ Nivel 5: en este nivel se busca una colaboración de negocios. El direccionamiento estratégico del proveedor debe ser tenido en cuenta, ya que el mejor proveedor en cuanto a tecnología y recursos humanos puede ser inadecuado si no considera el direccionamiento estratégico del negocio de su cliente.

Cuando se habla de integración, se debe establecer el tipo de trasferencia de conocimiento que realizará entre proveedor y cliente, lo que al final definirá el nivel de relación entre ellos. Entre los principales tipos de conocimientos a transferir se encuentran:

- Tecnológicos (Lin, 2003; Prencipe, 2000) respecto de habilidades y artefactos que pueden ser utilizados para desarrollar productos o servicios, incluyendo individuos, materiales, procesos, instalaciones, máquinas, equipos, teorías, principios, algoritmos y modelos entre otros.

- De mercado (Schulz, 2003), que pueden ser agrupados en conocimientos de negocios referente a experiencia con los clientes y la competencia, así como conocimientos institucionales referentes a capacidad de manejo de normatividad y valores en un determinado contexto institucional.

- De logística (Bronzo, 2004), asociado con el flujo de mercancías, servicios e información. Esto permite la trazabilidad de un producto así como su localización en tiempo real; de procesos (Prochno, 2004) relacionado con la producción de bienes, servicios y el lugar que se tiene dentro de la curva de aprendizaje.

- De conocimiento llamado relacional (Loureiro Rezende, Marques de Andrade Lima, y França Versiani, 2012) y que se refiere a la capacidad de formar, mantener y desarrollar relaciones con las combinaciones de recursos propios de los integrantes de la cadena de suministros, permitiendo satisfacer las necesidades mediante la utilización de las capacidades de los demás miembros de la cadena de suministros.

\section{Métodos de decisión multicriterio}

Existen diversos métodos de decisión multicriterio - Multiple Criteria Decision Making Methods (MCDM) que agrupan soluciones estadísticas, de programación matemática y de inteligencia artificial (Sung Ho y Krishnan, 2008), los cuales son utilizados para la evaluación de proveedores en vez del tradicionalmente considerado factor único de costo (Ruiz Torres, Mendoza, y Ablanedo Rosas, 2013).

Respecto de la utilización de estos métodos se puede encontrar trabajos como el de Ho, Xu y Dey (2010) quienes hacen una revisión de la literatura para resolver cuáles de los MCDM son prevalentemente utilizados y cuáles mejoras podrían hacerse a futuras aplicaciones de ellos. Dentro de los métodos empleados se destacan:

Análisis Envolvente de Datos (Data Envelopment Analysis-DEA) (Charnes, Cooper, y Rhodes, 1978), que utiliza una serie de modelos de programación lineal para estimar coeficientes de producción. Este método facilita la clasificación de los proveedores en eficientes o ineficientes inicialmente, pudiendo posteriormente aplicarse como lo hizo Jablonsky (2007) al construir un modelo que permitió obtener conjuntos de soluciones posibles que cumplieran con las restricciones impuestas dentro de la evaluación de eficiencia en las unidades de producción de un grupo de proveedores, lo que posteriormente complementó con técnicas multicriterio para dar contundencia a sus resultados. Reza (2006) aplicó también el método DEA para evaluar proveedores basado en múltiples criterios.

Analytic Network Process (ANP), se utiliza para obtener escalas de prioridad relativa de números absolutos a partir de juicios individuales, es implementado en el trabajo de Ustun y Demirtas (2008) para determinar la importancia de los criterios asociados con un proveedor, empleando esta información en un modelo de programación lineal entera mixta multi-objetivo y finalmente generar un conjunto de soluciones eficientes; Analytic Hierarchy Process (AHP) es un caso espe- 
cial de ANP que asume una independencia de los niveles jerárquicos más altos respecto de los más bajos y entre algunos elementos en un mismo nivel (Saaty, 2004), Fuzzy Set (Zadeh, 2011) utilizando comparaciones entre pares de elementos para la construcción de matrices mediante las cuales el modelo es capaz de establecer prioridades entre cada nivel y entre diferentes niveles (Gómez, Herrera Umaña, y Vinasco, 2008), siendo aplicable a situaciones en las que la fuente de imprecisión no es aleatoria ni posee límites definidos, por lo que se asigna grados intermedios entre 0 y 1 a cada uno de los objetos de estudio, permitiendo estructurar un problema complejo, de objetivos y sujetos múltiples de manera jerarquizada para posteriormente analizar cada nivel por separado, combinando resultados a medida que avanza el análisis (Sarache Castro, Castrillón Gómez, y Ortiz Franco, 2009); y Genetic Algorithm (Holland, 1992) que es planteado originalmente para programas computacionales que estudien la selección natural y teorías reproductivas naturales, siendo posteriormente utilizado por otros autores con algunas variaciones como en el trabajo de Sadeghieh, Dehghanbaghi, Dabbaghi, Barak (2012) quienes adicionan una teoría gris para cubrir la falta de información de los factores cualitativos y así encontrar una solución a la selección de proveedores.

En cuanto a establecer el mejor método, Oly et al. (2005), concluyeron que la selección de proveedores basada en tecnología es importante para las compañías que enfocan su producción en la flexibilidad de lanzamiento para los productos, mientras la calidad se convierte en estratégicamente importante cuando el productor está enfocado en la flexibilidad del volumen de producción. La administración de inventarios y hoja de ruta tecnológica son herramientas muy importantes para las estrategias de administración de proveedores con gran influencia en las tres formas de flexibilidad de la producción (Producto, lanzamiento y volumen). Es por eso fundamental la necesidad de que el productor comprenda claramente cuál de las flexibilidades requiere su operación, para así adoptar una estrategia de selección y administración de proveedores.

El método escogido para selección de proveedores debe ser objetivo y dinámico, para permitir una adecuada toma de decisiones, respecto de la continuidad o no de un proveedor, basado en elementos objetivos cuantificables y transparentes que no dejen duda a ninguna de las partes interesadas.
Uno de los métodos utilizables para la JOL de la FAC es el ya mencionado Proceso Analítico Jerárquico (AHP) el cual es una metodología matemática diseñada para resolver problemas de decisión cuando se tienen en consideración varios criterios, que reducen la subjetividad del proceso, dando resultados confiables para el responsable de la toma de decisiones. Está basado en el principio según el cual la experiencia y el conocimiento de las partes interesadas son tan importantes como los datos utilizados en el proceso. Las aplicaciones de ésta metodología relacionadas con la gestión de proveedores se puede evidenciar de manera práctica en trabajos de (Ghodsypour y O'Brien, 1998; Handfield, Walton, Sroufe, y Melnyk, 2002; Wang, Huang, y Dismukes, 2004; Chan y Kumar, 2007; Xia y Wu, 2007) entre otros.

El AHP es ilustrado de manera práctica por Osorio et al. (2008), mediante la aplicación del modelo en una empresa del sector servicios en Colombia, luego de una explicación general del modelo.

También, se encuentran modelos para selección de proveedores basados en análisis dimensional originalmente utilizado en física y que pueden ser resueltos mediante el uso de hojas de cálculo de común utilización, cuya aplicación práctica la muestran García, Alvarado y Maldonado (2013), lo que evidencia que la solución de los problemas no radica especialmente en la complicación del método utilizado sino más bien en la calidad y objetividad del análisis previo para la definición de los datos requeridos durante el procedimiento para selección de proveedores.

\section{Procedimiento general para gestión de proveedores}

Como se pudo evidenciar en los temas anteriores, lo que cualquier empresa o institución, incluida la FAC debe hacer inicialmente es definir categorías de suministros de acuerdo con su criticidad dentro de la organización, lo cual requiere disponibilidad de información exhaustiva y actualizada de los productos, que definitivamente aumenta el poder de negociación del equipo de compras, (Mettler y Rohner, 2009) para así mediante la aplicación de la filosofía del CM definir el nivel de integración (1 a 5) para establecer que tan simple o colaborativo debe ser el enfoque del sistema, buscando en aquellos proveedores de suministros críticos, aumentar valor agregado y no disminuir costos, ya que las ganancias vendrán de crear valor y no de reducir costos de abastecimiento (O'Keeffe y Fearne, 2002). 
A continuación se debe definir el objetivo que se pretende alcanzar por medio de la selección de proveedores para así establecer los criterios de evaluación dentro de los cuales pueden clasificarse las características de los productos o servicios de acuerdo con el nivel de integración requerido para la categoría a la que pertenezcan (Ghodsypour y O'Brien, 1998), lo cual de acuerdo con Kahraman, Cebeci y Ulukan (2003) puede no resultar fácil ya que generalmente se expresan las necesidades con conceptos cualitativos.

Los criterios que más frecuentemente se evalúan a los proveedores de acuerdo con diferentes autores, son reportados por Güneri, Ertay y Yücel (2011) relacionando el número de veces que el atributo ha sido citado por los autores, siendo los principales: calidad; capacidad técnica y tecnología usada; posición y situación financiera; instalaciones de producción y capacidad; entrega; flexibilidad y respuesta al cambio; y precio.

A continuación se aplica un método de calificación como los expuestos en los métodos de decisión multicriterio que generalmente ponderan los criterios para entonces utilizar un método de eliminación que satisfaga las reglas de selección y así tener uno o varios proveedores seleccionados (Eliacy Cavalcanti y Alexandre Tadeu, 2013). Siendo este último el paso final para aquellas categorías con niveles de integración 1 ó 2, pero apenas el inicio para niveles de integración mayores, donde se debe comenzar un proceso de desarrollo de estrategias por categoría robusto, que inicia con objetivos claros enfocados hacia la creación de valor, en el cumplimientos de los requerimientos de los clientes. Esto se materializa mediante acciones que incluyan el alineamiento de los objetivos y estrategias con los proveedores, el esfuerzo para innovación conjunta, integración del proveedor en el cumplimiento del proceso y todas aquellas que permitan mejorar las relaciones de trabajo. El horizonte de tiempo para las estrategias es, como fue mencionado anteriormente, de largo plazo, siendo para las compañías en general de entre tres y diez años (Monczka y Markham, 2014).

Finalmente, para todos los niveles de integración se debe establecer una evaluación periódica de proveedores que permita establecer el nivel de cumplimiento de las expectativas de desarrollo del proveedor, debiendo definir a su vez los criterios para cada categoría sin que esto signifique que los criterios de evaluación no puedan ser los mismos para todas las categorías. Para lo cual, se puede utilizar nuevamente cualquiera de los MCDM mencionados en este artículo. El procedimiento general se resume en la Figura 1.

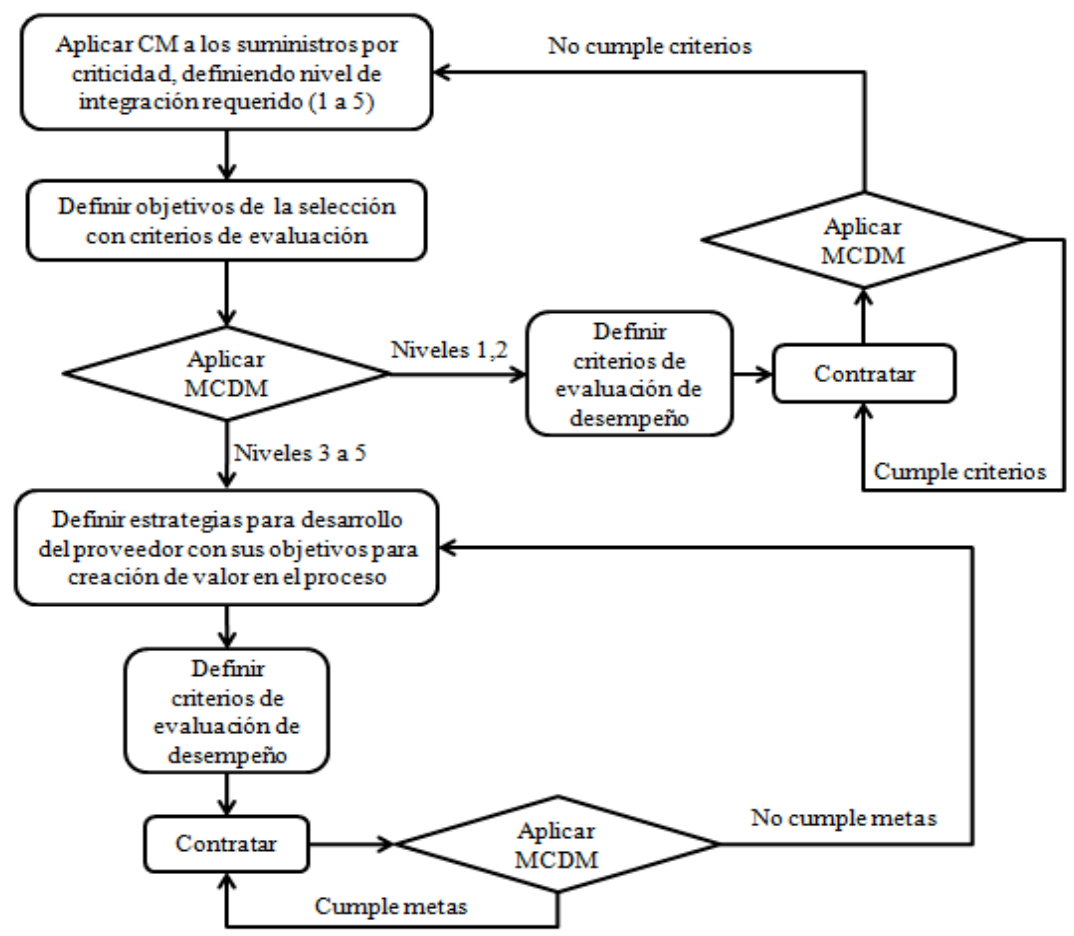

Figura 1. Procedimiento general para gestión de proveedores.

Fuente: Elaboración del autor. 


\section{Conclusiones}

De la revisión general de las mejores prácticas en gestión de proveedores a nivel mundial se pudo establecer que no existe un método único o esquema de aplicación universal, sino una combinación única para cada organización. Para esto se describió un procedimiento general, que permite gestionar proveedores para cumplir los objetivos y criterios en cuanto a tiempos de entrega, trazabilidad, calidad, costos y todos aquellos que la entidad considere relevantes.

El nivel de integración de un sistema tiene relación directa con el costo y el tiempo de implementación, por lo que debe buscarse un nivel de integración más alto para aquellos elementos que signifiquen mayor riesgo para el cumplimiento de los requerimientos y la misión del cliente. Para la FAC se debe realizar un análisis interno con enfoque CM para definir los materiales y proveedores que deben recibir la mayor atención y sobre los cuales se debe desarrollar relaciones colaborativas. Asimismo, establecer los stocks de seguridad que permitan compartir información logística sin poner en riesgo la seguridad nacional en caso de un paro en el flujo de los suministros.

La utilización de MCDM es solamente una herramienta que tendrá utilidad dependiendo del conocimiento y definición de variables que se haga al iniciar el procedimiento para la gestión de proveedores y no es un sistema de gestión por sí mismo. Los métodos de decisión multicriterio permiten evidenciar la transparencia en la ARP, al ser aplicados de manera objetiva y general.

\section{Referencias}

Aksoy, A., y Öztük, N. (2011). Supplier selection and performance evaluation in just-in-time production environments. Expert Systems With Applications.

Arroyo López, P. E., y Cárcamo Solis, M. d. (2009). Estudio comparativo sobre el desarrollo de proveedores en dos ramas industriales: automotriz y textil y de la confección. Contaduría y Administración, (228), pp. 105-126.

Barla, S. (2003). A case study of supplier selection for lean supply by using a mathematical model. Logistics Information Management, 16 (6), 451 doi:10.1108/09576050310503420.

Bronzo, M. (2004). Relacionamentos Colaborativos Em Redes de Suprimentos. RAE: Revista de Administração de Empresas, 44, pp. 61-73.

Chan, F. T., y Kumar, N. (2007). Global supplier development considering risk factors using fuzzy extended AHP-based approach. Omega, 35 (4), pp. 417- 431.
Charnes, A. A., Cooper, W. W., y Rhodes, E. E. (1978). Measuring the efficiency of decision making units. European Journal Of Operational Research, 2 (6), pp. 429-444.

Duanmu, J. L., y Fai, F. (2007). A Processual analysis of knowledge transfer: from foreign MNEs to Chinese suppliers. International Business Review, 16 (4), pp.449-473.

Dyer, J. H., y Chu, W. (2003). The role of trustworthiness in reducing transaction costos y improving performance, empirical evidence from United States, Japan, y Korea. Organization Science, 14 (1), pp. 57-68.

Eliacy Cavalcanti, L., y Alexandre Tadeu, S. (2013). Relationship management in a plastic part industry of automotive chain. Gest. Prod., 20 (4), pp. 889-911.

García Alcaraz, J. L., Alvarado Iniesta, A., y Maldonado Macías, A. A. (2013). Selección de proveedores basada en análisis dimensional. Contaduría y Administración, 58 (3), pp. 249-278.

Ghodsypour, S. H., y O'Brien, C. (1998). A decision support system for supplier selection using an integrated analytic hierarchy process and linear programming. International Journal of Production Economics, 56 (57), pp. 199-212.

Güneri, A., Ertay, T., y Yücel, A. (2011). An approach based on ANFIS input selection and modeling for supplier selection problem. Expert Systems With Applications, 3814907-14917.

Handfield, R., Walton, S. V., Sroufe, R., y Melnyk, S. A. (2002). applying environmental criteria to supplier assessment: a study in the application of the analytical hierarchy process. European Journal of Operational Research, 141 (1), pp. 70-88.

Hines, T. (2004). Supply Chain Strategies: Customer Driven and Customer Focused. Oxford: Butterworth-Heinemann.

Ho, W., Xu, X., y Dey, P. K. (2010). Multi-criteria decision making approaches for supplier evaluation and selection: A literature review. European Journal Of Operational Research, 1 (16).

Holland, J. H. (1992). Genetic algorithms. Scientific American, 267 (1), pp. 66.

Huang, S. H., y Keskar, H. (2007). Comprehensive and configurable metrics for supplier selection. International Journal of Production Economics, 2, 510.

Jablonsky, J. (2007). Measuring the efficiency of production units by AHP models. Journal of Mathematical and Computer Modelling, 27, pp. 10-13.

Kahraman, C., Cebeci, U., y Ulukan, Z. (2003). Multi-criteria supplier selection using fuzzy AHP. Logistics Information Management, 16 (6), 382.

Kannan, V. R., y Tan, K. C. (2002). Supplier selection and assessment: Their impact on business performance. Journal of Supply Chain Management, 38 (4), pp. 11-21.

Lin, B. (2003). Technology transfer as technological learning: a source of competitive advantage for firms with limited RyD resources. RyD Management, 33 (3), pp. 327-339.

Loureiro Rezende, S. F., Marques de Andrade Lima, W., y França Versiani, A. (2012). Evolução de Conhecimentos no Relacionamento Comprador-Fornecedor. Rev. Adm. Contemp (RAC), 16 (1), pp. 39-58.

Marques Vieira, L., Laureano Paiva, E., Beheregarai Finger, A., y Teixeira, R. (2013). Trust and Supplier-buyer Relationships: An 
Empirical Analysis. Brazilian Administration Review, 10 (3), pp. 263-280.

Mazzali, L., Machado Junior, C., y Jaciara Furlaneto, C. (2011). O nexo entre formalização e confiança na gestão da relação cliente-fornecedor. Gest. Prod, 18 (3), pp. 571-586.

Mettler, T., y Rohner, P. (2009). Supplier Relationship Management: A Case Study in the Context of Health Care. Journal of Theoretical and Applied Electronic Commerce Research, 4 (3), pp. 58-71.

Monczka, R. M., y Markham, W. J. (2014). The Future of Supply Management- Part I: Category Strategies and Supplier Management. Supply Chain Management Review, 11 (6), pp. 24-30.

O'Keeffe, M., y fearne, A. (2002). From commodity marketing to category management: Insights from the Waitrose category leadership program in fresh produce. Supply Chain Management: An International Journal, 7 (5), pp. 296-301.

Oly, N., Muhamad, J., Loo Cha, H., y Salleh, M. A. (2005). Supplier selection and management strategies and manufacturing flexibility. Journal Of Enterprise Information Management, $18(3), 330$.

Osorio Gómez, J., Herrera Umaña, M., y Vinasco, M. (2008). Modelo para la evaluación del desempeño de los proveedores utilizando AHP. Ingeniería y Desarrollo (23), 43.

Partida, B. (2012, july). The Value of Supplier Category Management. Supply Chain Management Review, pp. 2-84.

Pimentel Claro, D., Borin de Oliveira Claro, P., y Zylbersztajn, D. (2005). Relationship Marketing Strategies: When Buyer and Supplier Follow Different Strategies to Achieve Performance. Brazilian Administration Review, 2 (2), pp.17-34.

Prencipe, A. (2000). Breadth and depth of technological capabilities in CoPS: the case of the aircraft engine control system. (E. B.V., Ed.) Research Policy, 29 (7), pp. 895-911.

Pressey, A. D., Winklhofer, H. M., y Tzokas, N. X. (2009). Purchasing practices in small-to medium-sized enterprises: An examination of strategic purchasing adoption, supplier evaluation and supplier capabilities. Journal Of Purchasing And Supply Management, 15214-226.

Prochno, P. (2004). Learning Curve? Which One? Brazilian Administration Review, 1 (1), pp. 53-66.

Rashid, S., y Matilla, H. (2011). Study on the Scope and Opportunities of Category Management for Aligning the SupplierRetailer Business Strategy. South Asian Journal of Management, 18 (4), 62.

Reza, M. (2006). A data envelopment analysis approach based on total cost of ownership for supplier selection. Supply Chain Management, 19 (6), pp. 662-678.

Ruiz Torres, A. J., Ablanedo Rosas, J. H., y Ayala Cruz, J. (2012). Modelo de asignación de compras a proveedores considerando su flexibilidad y probabilidad de incumplimiento en la entrega. Estudios Gerenciales, 28 (122), pp. 29-48.

Ruiz Torres, A. J., Mendoza, A., y Ablanedo Rosas, J. H. (2013). Modelo para la planificación en la cadena de suministro: Selección y asignación a proveedores en el caso de lotes fijos. Ingeniería y Desarrollo, 31 (1), pp. 1-21.
Saaty, T. L. (2004). Fundamentals of the analytic network processDependence and feedback in decision-making with a single network. Journal of Systems Science and Systems Engineering, 13 (2), 129.

Sadeghieh, A. A., Dehghanbaghi, M. M., Dabbaghi, A. A., y Barak, S. S. (2012). A genetic algorithm based grey goal programming (G) approach for parts supplier evaluation and selection. International Journal Of Production Research, 50 (16), 4612.

Sarache Castro, W. A., Castrillón Gómez, Ó. D., y Ortiz Franco, L. F. (2009). Selección de proveedores: una aproximación al estado del arte. Cuadernos de Administración, 22 (38), pp. 145-167.

Schulz, M. (2003). Pathways of relevance: exploring inflows of knowledge into subunits of multinational corporations. Organization Science, 14 (4), 440-459.

Slack, N., Chambers, S., y Johnston, R. (2009). Administração da produção. Atlas, 747.

Sung Ho, H., y Krishnan, R. R. (2008). A hybrid approach to supplier selection for the maintenance of a competitive supply chain. Expert Systems With Applications, 2, 1303.

Ustun, O., y Demirtas, E. A. (2008). Multi-period lot-sizing with supplier selection using achivevement scalarizing functions. (E. S. Publishers, Ed.) Computers y Industrial Engineering, 54 (4), pp. 918-932.

Vereecke, A., y Muylle, S. (2006). Performance improvement through supply chain collaboration in Europe. International Journal of Operations y Production Management, 26 (11), pp. 1176-1198.

Wang, G., Huang, S. H., y Dismukes, J. P. (2004). Product-driven supply chain selection using integrated multi-criteria decision-making methodology. International Journal of Production Economics, 91 (1), pp. 1-15.

Xia, W., y Wu, Z. (2007). Supplier selection with multiple criteria in volume discount environments. (E. S. Publishers, Ed.) Omega, 35 (5), pp. 494-515.

Yeung, A. C. (2008). Strategic supply management, quality initiatives, and organizational performance. (A. P. Inc., Ed.) Journal of Operation Management, 26 (4), pp. 490-503.

Zadeh, L. A. (2011). Fuzzy Set Theory and Probability Theory: What is the Relationship? International Encyclopedia of Statistical Science, 1, pp. 563-566.

Zaheer, S., y Zaheer, A. (2006). Trust across borders. Journal of International Business Studies, 37 (1), pp. 21-29.

Para citar este artículo:

Arrazola, I. (2014). Mejores prácticas en gestión de proveedores a nivel mundial, aplicables al sistema de Administración de la Relación con el Proveedor (ARP) de la Jefatura de Operaciones Logísticas (JOL) de la Fuerza Aérea Colombiana (FAC). Ciencia y Poder Aéreo, Vol. 9 (1). Pp. 33-41 